FTUAM $94 / 8$

NIKHEF-H 94/14

UPRF 94/395

\title{
Shift versus no-shift in local regularizations of Chern-Simons theory
}

\author{
G. Giavarini \\ Libera Università della Bassa Ovest, Villa \\ Baroni 3, 43107 San Secondo P.se, Italy \\ and \\ Dipartimento di Fisica dell'Università di Parma, \\ Viale delle Scienze, I-43100 Parma, Italy \\ C. P. Martin \\ Departamento de Física Teórica, C-XI, Universidad Autónoma de Madrid \\ Cantoblanco, Madrid 28049, Spain \\ F. Ruiz Ruiz \\ NIKHEF-H, Postbus 41882, 1009 DB Amsterdam, The Netherlands
}

We consider a family of local BRS-invariant higher covariant derivative regularizations of $S U(N)$ Chern-Simons theory that do not shift the value of the ChernSimons parameter $k$ to $k+\operatorname{sign}(k) c_{V}$ at one loop. 
The problem of the uniqueness of the shift $k \rightarrow k+\alpha$ of the bare Chern-Simons parameter $k$ in perturbative quantization of Chern-Simons theory has been open to debate for some years. All explicit computations of $\alpha$ at one loop performed as yet [1-4] using local gauge invariant regulators have given the same result, namely $\alpha=\operatorname{sign}(k) c_{V}$. The uniqueness of the result and the very different nature of the regulators employed led to the conjecture [4] that gauge invariance, if preserved at the regularized level, chooses for the shift the value $\alpha=\operatorname{sign}(k) c_{V}$ at one loop. Further evidence for this conjecture came from the fundamental role that gauge invariance plays in establishing the connection between Chern-Simons theory and two-dimensional current algebra [1]. In addition, if the bare Chern-Simons parameter has the same meaning in perturbative quantization as in canonical quantization, the shift $\alpha=\operatorname{sign}(k) c_{V}$ is a necessary condition for the semiclassical evaluation of the partition function [3] to agree with the nonperturbative value computed using surgery techniques [1].

In ref. [4] we introduced a local biparametric family of gauge invariant regularizations based on higher covariant derivative actions. The actions we considered contained parity odd as well as parity even terms, and had a large-mometum behaviour dominated by parity even terms. We found that the shift $\alpha$ was independent of the family paramters and equal in all cases to $\alpha=\operatorname{sign}(k) c_{V}$. Very recently, the authors of ref. [5] have devised another family of regularization methods, still based on gauge-invariant higher covariant derivative actions, which allow for shifts different from $\alpha=\operatorname{sign}(k) c_{V}$. More precisely, for $k$ positive, they have obtained the three following types of radiative corrections $\alpha$ :

(i) $\alpha=c_{V}$, whenever the large-momentum leading term of the regularized action is parity even.

(ii) $\alpha=s c_{V}$, with $s=0,2$, whenever the large-momentum leading term of the regularized action is parity odd. The value of $s$ depending on the sign of such term.

(iii) $\alpha=r c_{V}$, with $r$ a real number, whenever the large-momentum leading 
term of the regularized action is a linear combination of parity even and parity odd terms. The value of $r$ depending on the coefficients of this linear combination.

The result in (i) agrees with all previous computations [1-3], and in particular with those in [4]. However, the results in (ii) and (iii) contradict the idea of a unique shift for all gauge invariant regulators, hence we think they deserve some consideration. They have been obtained using regularizations based on actions that contain nonlocal interactions, and is well known that theories whose actions contain nonlocal interactions do not generally have the same perturbative properties as theories whose actions only contain local interactions [6]. One could argue that the differences between regularizations involving nonlocal interactions and regularizations with only local interactions vanish as the regulator is removed. This, however, is not obvious since the unregularized theory is not finite by power counting. We thus find it necessary to reproduce the results in (ii) and (iii) by employing regularizations whose Feynman rules come from local actions. This way, any perverse effect due to the nonlocal structure of the regularization employed in ref. [5] can be ruled out. The purpose of this short note is to reproduce the type of one-loop radiative corrections in (ii) by using a fully BRS invariant regularization method whose action is local.

The classical Chern-Simons action for gauge group $S U(N)$ in the Landau gauge $\partial A^{a}=0$ is given by

$$
S=S_{\mathrm{CS}}+S_{\mathrm{GF}}
$$

where

$$
\begin{aligned}
& S_{\mathrm{CS}}=-\frac{i k}{4 \pi} \int d^{3} x \epsilon^{\mu \nu \rho}\left(\frac{1}{2} A_{\mu}^{a} \partial_{\nu} A_{\rho}^{a}+\frac{1}{3 !} f^{a b c} A_{\mu}^{a} A_{\nu}^{b} A_{\rho}^{c}\right) \\
& S_{\mathrm{GF}}=\int d^{3} x\left[-b^{a} \partial A^{a}+\left(J^{a \mu}-\partial^{\mu} \bar{c}^{a}\right)\left(D_{\mu} c^{a}\right)-\frac{1}{2} f^{a b c} H^{a} c^{b} c^{c}\right] .
\end{aligned}
$$

Here the notation is as in ref. [7], and for convenience we choose $k$ to be positive. To regularize the theory, we add to $S$ the following higher covariant derivative 
terms:

$$
S_{\Lambda}=S+\frac{k}{4 \pi} \int d^{3} x\left[\frac{1}{4 \Lambda} F_{\mu \nu}^{a} F^{a \mu \nu}-\frac{i v}{2 \Lambda^{2}} \epsilon^{\mu \nu \rho} F_{\mu \sigma}^{a}\left(D_{\nu} F_{\rho}^{\sigma}\right)^{a}\right]
$$

with $\Lambda$ the higher covariant derivative mass and $v$ an arbitrary real parameter. For all $v$ different from zero, the large-momentum leading term in $S_{\Lambda}$ is parity odd, so eq. (1) provides a family of local actions satisfying the parity requirement in (ii) above. The adding of the higher covariant derivative terms in eq. (1) does not completely regularize the theory, since there is still a finite number of Feynman diagrams divergent by power counting. To regularize these diagrams, we use 't Hooft-Veltman's dimensional regularization prescription for theories involving parity violating objects [8] (see ref. [7] for details). Since this prescription manifestly preserves gauge invariance and the action $S_{\Lambda}$ is BRS invariant, we end up with a local BRS-invariant regularization method which combines the mass $\Lambda$ and the dimensional regulator $D$. Here we are concerned with radiative corrections to the bare Chern-Simons parameter, so we will be working in a renormalization scheme characterized by $k_{\text {renormalized }}=k_{\text {bare }}=k$, or in other words by the renormalization constant for $k$ being simply $Z_{k}=1$. We define renormalized Chern-Simons theory in this scheme as the limit $\Lambda \rightarrow \infty$ of the limit $D \rightarrow 3$ of the dimensionally regularized theory whose classical action is $S_{\Lambda}$.

We recall that up to one loop the local part of the renormalized effective action, obtained as a solution of the BRS identities, can be cast as [7] [9]

$$
\Gamma^{\mathrm{loc}}=-\frac{i(k+\alpha)}{4 \pi} S_{\mathrm{CS}}+\int d^{3} x\left\{-b^{a} \partial A^{a}+\Delta\left[\beta\left(J^{a \mu}-\partial^{\mu} \bar{c}^{a}\right) A_{\mu}^{a}-(1+\gamma) H^{a} c^{a}\right]\right\}
$$

Here $\alpha, \beta$ and $\gamma$ are coefficients of order $\hbar$, and $\Delta$ is the Slavnov-Taylor operator for the theory [7] [9]. The effective action contains two types of radiative corrections: gauge invariant radiative corrections, labeled by $\alpha$, which make the shift, and gauge dependent radiative corrections, labeled by $\beta$ and $\gamma$. The coefficients $\alpha, \beta$ and $\gamma$ can be uniquely determined from the vacuum polarization 
tensor $\Pi_{\mu \nu}^{a b}$, the ghost self-energy $\Omega^{a b}$ and the $H c c$-vertex $V^{a b c}$. All we have to do then is to compute the limit $\Lambda \rightarrow \infty, D \rightarrow 3$ of these three Green functions. This is done by following the same steps as in ref. [4], the only difference being that the $D$-dimensional gauge field propagator now reads

$$
D_{\mu \nu}^{a b}(q)=\frac{4 \pi}{k} \frac{\Lambda^{2} \delta^{a b}}{q^{2} P\left(q^{2}, \Lambda ; v\right)}\left[\left(\Lambda^{2}+v q^{2}\right) \epsilon_{\mu \rho \nu} q^{\rho}+\Lambda\left(q^{2} g_{\mu \nu}-q_{\mu} q_{\nu}\right)\right]
$$

where $P\left(q^{2}, \Lambda ; v\right)$ has the form *

$$
P\left(q^{2}, \Lambda ; v\right)=\left(\Lambda^{2}+v q^{2}\right)^{2}+\Lambda^{2} q^{2}
$$

and $\epsilon_{\mu \rho \nu}$ in $D$ dimensions is understood in the 't Hooft-Veltman sense [7]. Afer some calculations, we obtain

$$
\Pi_{\mu \nu}^{a b}(p)=c_{V} J(v) \epsilon_{\mu \rho \nu} p^{\rho} \delta^{a b} \quad \Omega^{a b}(p)=-\frac{c_{V}}{k} I(v) p^{2} \delta^{a b} \quad V^{a b c}\left(p_{1}, p_{2}\right)=0
$$

where $p^{\mu}, p_{1}^{\mu}$ and $p_{2}^{\mu}$ are external momenta, and $J(v)$ and $I(v)$ are given by

$$
\begin{aligned}
& J(v)=-\frac{2}{3 \pi} \int_{-\infty}^{\infty} d t \frac{3 v^{3} t^{6}-8 v^{2} t^{4}-(13 v+5) t^{2}-2}{P^{2}\left(t^{2}, 1 ; v\right)} \\
& I(v)=\frac{2}{3 \pi} \int_{-\infty}^{\infty} d t \frac{1}{P\left(t^{2}, 1 ; v\right)} .
\end{aligned}
$$

Note that in the renormalization scheme we are working there are no restrictions on the renormalization constants $Z_{\phi}$ for the fields $\phi=A_{\mu}^{a}, b^{a}, \bar{c}^{a}, \ldots$ To account for this arbitrariness, we replace the fields $\phi$ in the effective action $(2)$ with $\left(1+z_{\phi}\right) \phi$,

\footnotetext{
$\star$ Note that $P\left(q^{2}, \Lambda ; v\right)$ is positive definite for all $v$ and all $q^{\mu}$, which ensures that power counting holds.
} 
where the $z_{\phi}$ 's are abitrary coefficients of order $\hbar$ satisfying the BRS identites $z_{A}+z_{b}=0$ and $z_{A}+z_{\bar{c}}=z_{c}+z_{H}$. The results in eq. (3) then imply that

$$
\alpha=c_{V}[J(v)-2 I(v)] \quad \beta+z_{A}=\frac{4}{3} \frac{c_{V}}{k} I(v) \quad \gamma+2 z_{c}+z_{H}=0 .
$$

Some simple algebra to combine $J(v)-2 I(v)$ into a single integral and Cauchy's residue theorem finally lead to the following result for $\alpha$ :

$$
\alpha=\left\{\begin{array}{cc}
0 & \text { if } v>0 \\
2 c_{V} & \text { if } v<0
\end{array}\right.
$$

We thus see that our regularization method, based on actions with only local interactions, reproduces the one-loop shifts in (ii) above.

Here we have calculated the shift $\alpha$ for $k>0$. The value of $\alpha$ for $k<0$ can be retrieved from our previous computations in the following way. Assume that we change the sign of the term $F^{2} / 4 \Lambda$ in eq. (1) and that we replace $k$ with $\operatorname{sign}(k)|k|$. Radiative contributions to the local effective action $\Gamma^{\text {loc }}$ involving an odd number of epsilons then pick an overall factor $\operatorname{sign}(k)$. This implies in particular that the shift $\alpha$ for $k<0$ becomes $\operatorname{sign}(k)$ times the value of $\alpha$ for $k>0$.

It is very easy to see that the type of shifts (iii) can not be reproduced using a local higher covariant derivative term. Indeed, some simple dimensional analysis shows that a local higher covariant derivative parity-odd term never has the same mass dimension as a local higher covariant derivative parity-even term. Hence, both terms can not be linearly combined into a single local term, which is the requirement in (iii).

Acknowledgements: FRR was supported by FOM, The Netherlands. The authors also acknowledge partial support from CICyT, Spain. 


\section{References}

1. E. Witten, Commun. Math. Phys. 121 (1989) 351.

2. L. Alvarez-Gaumé, J.M.F. Labastida and A.V. Ramallo, Nucl. Phys. 334B (1990) 103.

M. Asorey and F. Falceto, Phys. Lett. 241B (1990) 31.

C. P. Martin, Phys. Lett. 241B (1990) 513.

G. P. Korchemsky, Mod. Phys. Lett. 6A (1990) 727.

D. Birmingham, R. Kantwoski and M. Rakowski, Phys. Lett. 251B (1990) 367.

G. Leibbrandt and C. P. Martin, Nucl. Phys. 377B (1992) 593.

3. D.S. Freed and R.E. Gompf, Commun. Math. Phys. 141 (1991) 79.

4. G. Giavarini, C. P. Martin and F. Ruiz Ruiz, Phys. Lett. 314B (1993) 328.

5. M. Asorey, F. Falceto, J. L. López and G. Luzón, Universality and Ultraviolet Regularizations of Chern-Simons Theory, DFTUZ 93.10 preprint.

6. D. A. Eliezer and R. P. Woodard, Nucl. Phys. 325B (1989) 389.

7. G. Giavarini, C.P. Martin and F. Ruiz Ruiz, Nucl. Phys. 381B (1992) 222.

8. J.C. Collins, Renormalization (Cambridge University Press, Cambridge, 1984).

9. G. Giavarini, C. P. Martin and F. Ruiz Ruiz, Phys. Rev. 47D (1993) 5536. 